\title{
Atorvastatin ameliorates early brain injury after subarachnoid hemorrhage via inhibition of AQP4 expression in rabbits
}

\author{
JUN-HUI CHEN $^{1 *}$, LI-KUN YANG $^{1 *}$, LEI CHEN ${ }^{1}$, YU-HAI WANG ${ }^{1}$, YUN WU $^{2}$, \\ BING-JIE JIANG ${ }^{1}$, JIE ZHU ${ }^{1}$ and PEI-PEI LI ${ }^{1}$ \\ ${ }^{1}$ Department of Neurosurgery, 101st Hospital of PLA (Wuxi Taihu Hospital), \\ Clinical Medical School of Anhui Medical University, Wuxi, Jiangsu 214044; ${ }^{2}$ Department of Anesthesia, \\ The First Affiliated Hospital of Gannan Medical College, Jiangxi 341000, P.R. China
}

Received August 12, 2015; Accepted February 11, 2016

DOI: $10.3892 /$ ijmm.2016.2506

\begin{abstract}
The therapeutic effects of atorvastatin on early brain injury (EBI), cerebral edema and its association with aquaporin 4 (AQP4) were studied in rabbits after subarachnoid hemorrhage (SAH) using western blot analysis and the dry-wet method. Seventy-two healthy male New Zealand rabbits weighing between 2.5 and $3.2 \mathrm{~kg}$ were randomly divided into three groups: the SAH group $(n=24)$, sham-operated group $(n=24)$ and the $\mathrm{SAH}+$ atorvastatin group $(n=24)$. A double SAH model was employed. The sham-operated group were injected with the same dose of saline solution, the $\mathrm{SAH}+$ atorvastatin group received atorvastatin $20 \mathrm{mg} / \mathrm{kg} /$ day after $\mathrm{SAH}$. All rabbit brain samples were taken at $72 \mathrm{~h}$ after the SAH model was established successfully. Brain edema was detected using the dry-wet method after experimental SAH was induced; AQP4 and caspase-3 expression was measured by western blot analysis, and neuronal apoptosis was detected by terminal deoxynucleotidyl transferase-mediated dUTP nick end labelling (TUNEL) staining at $72 \mathrm{~h}$ after SAH. The results indicated that brain edema and injury appeared soon after SAH, while brain edema and EBI were ameliorated and increased behavior scores were noted after prophylactic use of atorvastatin. Compared with the SAH group, the level of AQP4 and the cerebral content of water was significantly decreased $(\mathrm{P}<0.01)$ by atorvastatin, and TUNEL staining and studying the expression of caspase-3 showed that the apoptosis of neurons was reduced markedly both in the hippocampus and brain cortex by atorvastatin. The results suggest that atorvastatin ameliorated brain edema and
\end{abstract}

Correspondence to: Dr Yu-Hai-Wang, Department of Neurosurgery, 101st Hospital of PLA (Wuxi Taihu Hospital), Clinical Medical School of Anhui Medical University, Wuxi, Jiangsu 214044, P.R. China

E-mail: wangyuhai67@126.com

${ }^{*}$ Contributed equally

Key words: subarachnoid hemorrhage, atorvastatin, aquaporin 4, brain edema
EBI after SAH, which was related to its inhibition of AQP4 expression. Our findings provide evidence that atorvastatin is an effective and well-tolerated approach for treating SAH in various clinical settings.

\section{Introduction}

Spontaneous subarachnoid hemorrhage ( $\mathrm{SAH})$ is a very common cerebrovascular condition and affects 9/100,000 in the Western world. Although it accounts for only $5 \%$ of all strokes, it is a devastating neurological condition with generally poor outcome and high levels of mortality and morbidity (1-3). Early brain injury (EBI), acute hydrocephalus, delayed cerebral vasospasm (CVS) and cerebral infraction play an important role in poor prognosis after SAH. Even though a large number of previous studies on SAH have focused mainly on delayed CVS $(7,10,12,13,18,22)$, Laskowitz et al (4) found that the functional outcome was not improved even if the angiographic vasospasm was reversed. MacDonald et al (5) confirmed that clazosentan, an endothelial receptor antagonist, ameliorated CVS after SAH significantly, but it did not improve the outcome.

EBI occurs within $72 \mathrm{~h}$ after SAH, Sehba et al (6) have reported that it is more common than CVS in cases with poor outcomes. The pathophysiological mechanisims of EBI after SAH include increased intracranial pressure, oxidative stress leading to inflammation, blood-brain barrier (BBB) disruption and cerebral ischemia. All of these factors lead to neuronal cell death and brain edema, and brain edema also increases intracranial pressure and aggravates EBI (7-9). Certain studies have shown that EBI, which manifests as early cortical feedback depolarization waves, cortical spreading depression and impaired neurovascular coupling, plays a crucial important role in neurological deterioration after SAH (10-12).

Statins, inhibitors of the 3-hydroxy-3-methylglutaryl coenzyme A (HMG-CoA) reductase, are widely used in cardiovascular medicine as cholesterol-lowering drugs and it has also been suggested to exert pleiotropic effects, including exerting anti-inflammatory (13), anti-oxidative stress (14), and anti-CVS effects $(7,15)$, as well as inhibiting platelet aggregation $(16,17)$. However, the full effect and specific pathophysiological mechanisms of statins on EBI after SAH remain not fully understood. Atorvastatin has been shown to act as a potent statin and 
inhibitor of HMG-CoA, and Cheng et al (7) have reported that atorvastatin exerted neuroprotective- and anti-CVS effects in experimental rat models of SAH.

The aim of the present study was to evaluate the effect of atorvastatin on SAH-induced EBI, and then to demonstrate that atorvastatin attenuates EBI and brain edema in experimentally induced SAH via the inhibition of aquaporin 4 (AQP4) expression.

\section{Materials and methods}

Animals and drugs. The animals used and the care protocols were approved by the Animal Care and Use Committee of Anhui Medical University (Wuxi, China) and all experiments conformed to the Guide for the Care and Use of Laboratory Animals by the National Institute of Health. All 72 New Zealand white rabbits used in the present study were purchased from the Animal Central of Taihu Hospital (Wuxi, China). They were raised in a comfortable room with normal levels of atmospheric moisture and fed with a standard diet at the Animal Center of Taihu Hospital (Wuxi, China) for 10 days before the experiments. The temperature of the feeding room and operation room was maintained at approximately $22^{\circ} \mathrm{C}$. We chose a dosage of $20 \mathrm{mg} / \mathrm{kg} /$ day atorvastatin. Atorvastatin was orally administered by gastric gavage once daily for 3 days before and also at $22 \mathrm{~h}$ after SAH to maintain drug levels. We assessed the neurologic deficits at $24 \mathrm{~h}$ after SAH and rabbits were sacrificed immediately after the neurological evaluation. Atorvastatin was obtained from Pfizer (Jiangsu, China).

Experimental design. All adult male New Zealand white rabbits weighing between 2.5 and $3.2 \mathrm{~kg}$ were assigned randomly to three groups: i) Sham-operated group ( $\mathrm{n}=24)$, ii) SAH group $(n=24)$, and the SAH + atorvastatin group $(n=24)$. For the rabbits in the $\mathrm{SAH}+$ atorvastatin group $(\mathrm{n}=24)$, atorvastatin $(20 \mathrm{mg} / \mathrm{kg} / \mathrm{day})(7)$ was administered immediately after the first blood injection and was continued every 24 for $72 \mathrm{~h}$. The sham-operated rabbits underwent the same operation to induce SAH as the SAH group, but the sham-operated group was injected with saline solution. All rabbits were sacrificed on day 3. Eight rabbits in each group were sacrificed using the perfusion-fixation method. The hippocampus was collected for terminal deoxynucleotidyl transferase-mediated dUTP nick end labelling (TUNEL) staining. Eight rabbits were sacrificed in order to evaluate the brain water content. The other eight rabbits were exsanguinated and decollated in each group. The brain tissue was removed and frozen in a deep cryogenic refrigerator for biochemical studies. Before rabbits were sacrificed, serums were sampled in two copies to detect endothelin-1 (ET-1).

SAH model. Experimental SAH was induced according to the two-hemorrhage model, as previously described, using rabbits (7). The rabbits were anesthetized via auricular marginal vein injection of $10 \%$ chloral hydrate $(2.5 \mathrm{ml} / \mathrm{kg})$. Life signs were maintained at a stable level, and we inserted a 23-gauge butterfly needle into the cisterna magna. After $1 \mathrm{ml}$ cerebrospinal fluid (CSF) was released, then $2 \mathrm{ml}$ non-heparinized fresh autologous auricular artery blood was injected into the cisterna magna for 1 min under strict aseptic conditions. To make blood flow easily from the cisterna magna
Table I. Behavior scores.

\begin{tabular}{llc}
\hline Category & \multicolumn{1}{c}{ Behavior } & Score \\
\hline \multirow{2}{*}{ Appetite } & Finished meal & 0 \\
& Left meal unfinished & 1 \\
& Scarcely ate & 2 \\
Activity & Active, squeaking or standing & 0 \\
& Lying down, will stand and walk & \\
& with some stimulation & 1 \\
& Almost always lying down & 2 \\
Deficits & No deficits & 1 \\
& Unable to walk due to ataxia or paresis & \\
& Impossible to walk and stand & 2 \\
& due to ataxia and paresis & \\
\hline
\end{tabular}

to the basilar cistern, all rabbits were kept in a $30^{\circ}$ head-down position for $30 \mathrm{~min}$. They were returned to the feeding room after recovering from anesthesia. The second injection was administered after $48 \mathrm{~h}$ in the same manner as the first.

Behavior scoring. All rabbits behavior scores were recorded by the same independent observer who was blinded to the study. We used a previously modified scoring table (Table I) to evaluate the neurological function every day, as previously described (18).

Measuring brain water content. The entire brain was removed at $72 \mathrm{~h}$ and weighed immediately (wet weight) and again after being dried in an oven at $100^{\circ} \mathrm{C}$ for $24 \mathrm{~h}$ (dry weight). The percentage of brain water content was calculated as follows: (wet weight-dry weight)/wet weight $\mathrm{x} 100 \%$. The number of rabbits used for this in each group was $\mathrm{SAH}+$ atorvastatin group $(n=8)$, SAH group $(n=8)$ and sham-operated group $(n=8)$.

Perfusion-fixation. Eight rabbits in each group scheduled for sacrifice were anesthetized with an injection of $10 \%$ chloral hydrate $(4 \mathrm{ml} / \mathrm{kg})$ to the uricular marginal veins. The chests of the rabbits were quickly opened for intubation with a cannula in the left ventricle, and the right atrium was opened. Perfusion began with $1,500 \mathrm{ml}$ physiological phosphate buffer solution $(0.01 \mathrm{M}=\mathrm{PBS}, \mathrm{pH} 7.3)$ at $37^{\circ} \mathrm{C}$, and was then followed by $1,000 \mathrm{ml} 10 \%$ buffered formaldehyde under $120 \mathrm{~cm} \mathrm{H}_{2} \mathrm{O}$ perfusion pressure. After perfusion, the whole brain and the brain tissue were removed and stored in formalin.

TUNEL staining and cell counting. A TUNEL staining kit (Roche Inc., Basel, Switzerland) was used to stain brain sections; the TUNEL-positive cells were indicated by fluorescein-dUTP with dNTP or peroxidase (POD) with 3-3' diaminobenzidine (DAB). This was undertaken according to the manufacturer's instructions for the in situ Apoptosis Detection kit (Roche Inc., Mannheim, Germany) as previously described (18). The negative control was similarly performed but TUNEL reaction mixture was omitted. Cells exhibiting nuclear condensation/ fragmentation and apoptotic bodies in the absence of cyto- 
plasmic TUNEL reactivity and brown staining of nuclei were considered apoptotic cells. Apoptotic cells were confirmed with the help of a pathologist blinded to the grouping. The number of TUNEL-positive cells in each region was counted in a highpowered field (x400) by an investigator who was blinded to the studies, and expressed as number $/ \mathrm{mm}^{2}$. The number of animals used in each group was as follows: $\mathrm{SAH}+$ atorvastatin $(n=8)$, SAH $(n=8)$, and sham-operated $(n=8)$.

Enzyme-linked immunosorbent assay (ELISA). At day 3 after surgical intervention, blood samples were collected from anesthetized animals, and analyzed for ET-1 expression levels using an ELISA kit (Abcam, Cambridge, UK) specific for rabbits. To collect the plasma, the homogenates were centrifuged at $3,000 \mathrm{~g} / \mathrm{min}$ for $15 \mathrm{~min}$, and the supernatant was assayed for the protein concentration of ET-1 (Abcam), in accordance with the manufacturer's instructions. The concentrations $(\mathrm{pg} / \mathrm{ml})$ were determined based on a standard curve, prepared using a known set of serial dilutions of standard proteins by BCA assay. The number of animals used in the ELISA and histological study are as follows: sham-operated group $(n=8)$, SAH group $(n=8)$, and $\mathrm{SAH}+$ atorvastatin group $(\mathrm{n}=8)$.

Western blot analysis. The method of western blot analysis for evaluating AQP4 and caspase-3 has been described previously (19). The samples (20 $\mu \mathrm{g}$ total protein) were separated by sodium dodecyl sulfate polyacrylamide gel for electrophoresis with $10 \%$ polyacrylamide gel. The following primary antibodies were used: rabbit anti-AQP4 (1:2,000; ab46182) and anti-caspase-3 (1:500; ab2171) antibody (Abcam). The glyceraldehyde-3-phosphate dehydrogenase (GAPDH) (diluted in 1:6,000; Sigma-Aldrich, Inc., St. Louis, MO, USA) was used as a loading control. After incubation with the primary antibodies, the nitrocellulose membranes were washed with $0.01 \mathrm{M}$ TBST dilution and incubated with appropriate horseradish peroxidase-labeled secondary antibodies (1:1,000; Santa Cruz Biotechnology Inc., Santa Cruz, CA, USA) using 1\% non-fat milk in TBST for $1 \mathrm{~h}$ at room temperature. After two rinses and four washes with TBST, the membranes were incubated in ECL (Amersham, Little Chalfont, UK) reagent for HRP (60 sec) and exposed to autoradiography film for visualization of the bands. The results were quantified using Quantity One Software (BioRad Laboratories, Hercules, CA, USA). The number of animals used in each group was SAH + atorvastatin $(n=8)$, SAH $(n=8)$ and Sham $(n=8)$.

Statistical analysis. All data are presented as the means \pm SD. SPSS 14.0 (Anhui Medical University, SPSS Inc., Anhui, China) was used for statistical analysis of the data. Differences between the two groups were analyzed using a two-tailed unpaired Student's t-test. The differences among multiple groups were assessed using one-way analysis of variance (one way ANOVA). Ranked data between the two groups were evaluated using the rank sum test. A P-value $<0.05$ was considered to indicate a statistically significant difference.

\section{Results}

General observations. In the interval and by the end of the experiment, there was no obvious difference in blood pres-

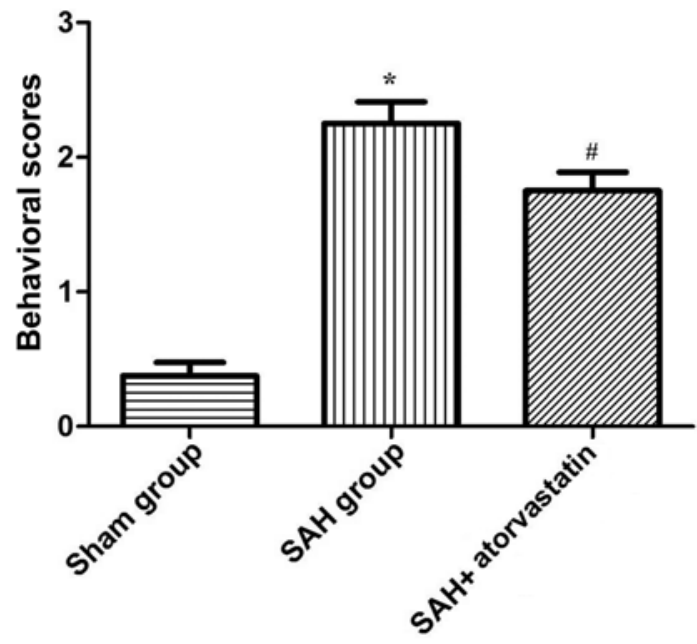

Figure 1. Graphs showing the behavior scores of the groups $(n=24)$. Bar graphs represent average values of behavior scores, and data are represented as the means + SD. Sham-operated group vs. subarachnoid hemorrhage $(\mathrm{SAH})$ group and $\mathrm{SAH}+$ atorvastatin group, ${ }^{*} \mathrm{P}<0.01$ and ${ }^{\#} \mathrm{P}<0.01$; $\mathrm{SAH}+$ atorvastatin group vs. $\mathrm{SAH}$ group, ${ }^{\#} \mathrm{P}<0.05$.

sure, injected arterial blood gas data, body weight and blood pressure. The mortality of the SAH group was $33.3 \%(8 / 24)$, $20.8 \%(5 / 24)$ in the SAH + atorvastatin treated group and none in the sham-operated group $(0 / 24)$. The mortality of $\mathrm{SAH}+$ atorvastatin treated group was significantly lower than in the SAH group $(\mathrm{P}<0.05)$. In the process of constructing the model, we removed the rabbits which died, or the rabbits which dif not meet the requirements, and added new rabbits randomly to ensure the number of animals was maintained in each group (all data not shown).

Behavior scoring. The behavior scores of rabbits in the SAH group and $\mathrm{SAH}+$ atorvastatin group were both significantly higher than the sham-operated group $(\mathrm{P}<0.01$; according to ANOVA), but the behavior scores in the SAH + atorvastatin group was significantly lower $(\mathrm{P}<0.05$; ANOVA) (Fig. 1) than that in the SAH group after $72 \mathrm{~h}$. Thus, our results showed that atorvastatin improves neurological functional after $\mathrm{SAH}$ in experimental rabbits.

Brain water content. The brain water content of the $\mathrm{SAH}+$ atorvastatin group and SAH group all significantly increased (80.130.60 vs. 79.080.36, $\mathrm{P}<0.05 ; 82.160 .41$ vs. 79.080.36, $\mathrm{P}<0.05)$ compared to the sham-operated group at $72 \mathrm{~h}$ after SAH. Brain water content was decreased significantly by atorvastatin treatment as compared with that of the SAH group (80.130.60 vs. 82.160.41, $\mathrm{P}<0.05$ ) (Fig. 2).

TUNEL staining and cell death assay. There were almost no TUNEL-positive cells detected in the sham-operated group animals. TUNEL-positive cells were significantly increased in the hippocampus of rabbits at $72 \mathrm{~h}$ after SAH. TUNEL-positive cells significantly decreased in the $\mathrm{SAH}+$ atorvastatin treatment group $(\mathrm{P}<0.05)$ (Fig. 4).

Protein ET-1 expression. In this study, we performed ELISA to examine the changes in protein expression of the vasoconstrictor 


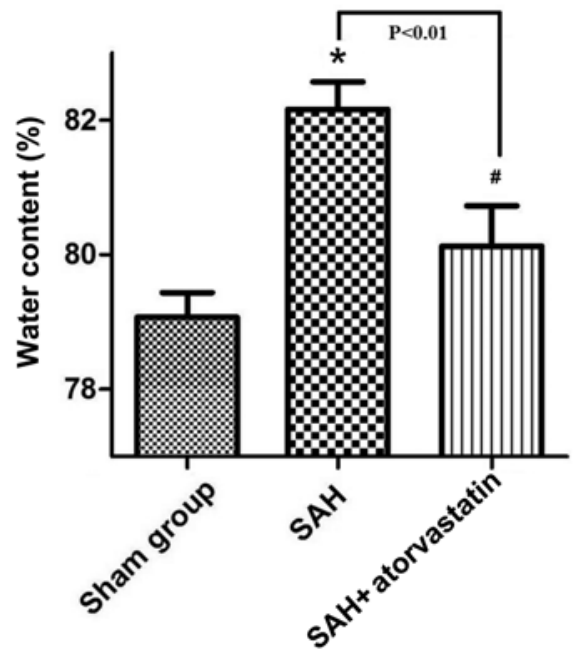

Figure 2. Water content of the brain tissues of rabbits at $72 \mathrm{~h}$ following subarachnoid hemorrhage (SAH). Graph showing the brain water content of the groups $(n=8)$. Bar graph represents averaged values of the brain water content; data are represented as the means $+\mathrm{SD}$. Sham-operated group vs. SAH group and $\mathrm{SAH}+$ atorvastatin group, ${ }^{*} \mathrm{P}<0.01$ and ${ }^{\# \mathrm{P}}<0.01$; $\mathrm{SAH}+$ atorvastatin group vs. $\mathrm{SAH}$ group, ${ }^{\#} \mathrm{P}<0.01$.

ET-1, to determine the effect of atorvastatin on cerebral edema after SAH. As is shown in Fig. 3, compared with the sham-operated group, ET-1 expression was markedly increased in all SAH rabbits (SAH group, $85.24+6.25$ vs. $39.72+4.67 \mathrm{pg} / \mathrm{ml}$, $\mathrm{P}<0.01 ; \mathrm{SAH}+$ atorvastatin, $62.92 \pm 7.27$ vs. $39.72 \pm 4.67 \mathrm{pg} / \mathrm{ml}$, $\mathrm{P}<0.01)$. After atorvastatin treatment, the elevation of plasma ET-1 concentration was significantly lower than the SAH group $(62.92 \pm 7.27$ vs. $85.24 \pm 6.25 \mathrm{pg} / \mathrm{ml}, \mathrm{P}<0.01)$ (Fig. 3).

AQP4 protein expression. In the present study, AQP4 protein expression was evaluated in the brain cortex; the level of cleaved AQP4 was evaluated by western blot analysis after SAH ( $\mathrm{P}<0.05$ vs. sham-operated group). Atorvastatin clearly reduced the expression of cleaved AQP4 $(\mathrm{P}<0.05$ vs. SAH rabbits) after SAH (Fig. 5).

Caspase- 3 protein expression. Caspase- 3 expression in the hippocampus was detected by western blot analysis in order to observe neuronal apoptosis at $72 \mathrm{~h}$ after SAH. SAH induced a marked increase in caspase- 3 in the hippocampus, whereas the level of caspase-3 decreased markedly in the $\mathrm{SAH}+$ atorvastatin group $(\mathrm{P}<0.05)$ (Fig. 6).

\section{Discussion}

Certain randomized, controlled clinical trials $(15,20,21)$ have investigated the effects of acute statin post-treatment on SAH, and mainly noted delayed CVS and outcomes. By contrast, previous studies (22-24) have found that the beneficial effects of statins on functional outcomes and delayed CVS are controversial, or even invalid. Therefore, the effects of acute statin post-treatment on SAH were questionable. However, a large number of experiments $(7,9,14,15,25,26)$ have confirmed that statins prevent delay CVS, ameliorate EBI and improve the outcome of patients after SAH. However, the mechanism of EBI remained not fully understood. In the present study,

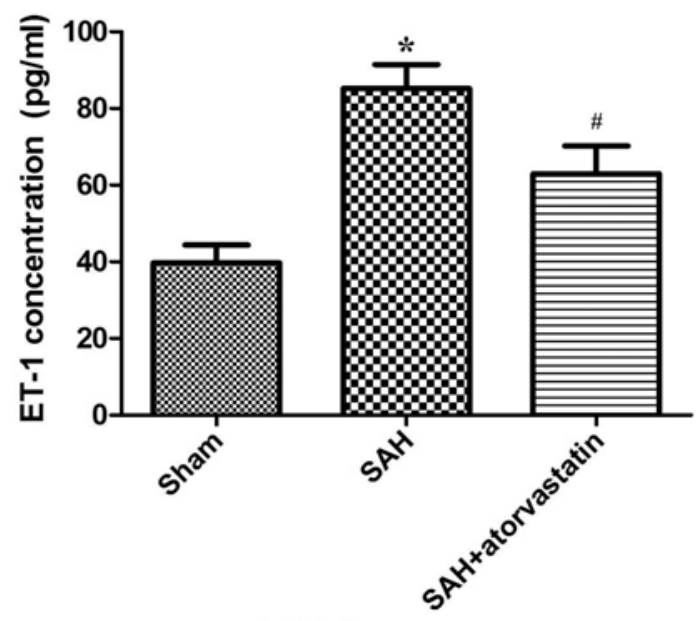

Figure 3. Enzyme-linked immunosorbent assay (ELISA)-based examination of the effect of atorvastatin on endothelin-1 (ET-1) in the plasma of the rabbits at $72 \mathrm{~h}$ following subarachnoid hemorrhage (SAH). Graph showing the ET-1 protein levels of the groups $(n=8)$. Bar graph represents averaged values of ET-1 concentration; data are represented as the means $+\mathrm{SD}$. Sham-operated group vs. SAH group and $\mathrm{SAH}+$ atorvastatin group, ${ }^{*} \mathrm{P}<0.01$ and ${ }^{\#} \mathrm{P}<0.01$; $\mathrm{SAH}+$ atorvastatin group vs. SAH group, ${ }^{\#} \mathrm{P}<0.01$.

we demonstrated that atorvastatin ameliorated EBI and brain edema after experimental SAH. Inhibition of AQP4 was also observed after treatment with atorvastatin.

$\mathrm{EBI}$ is one of the most important causes of delayed cerebral dysfunction; Broderick et al (27) firstly proposed the concept of EBI after SAH, and key pathological hallmarks of EBI after SAH were thought to be neuronal cell death and brain edema. Claassen et al (28) demonstrated that $8 \%$ of patients had brain edema after bleeding, according to CT examination, and $12 \%$ exhibited brain edema 6 days after bleeding. Cerebral edema increased intracranial pressure and decreased cerebral blood flow. The mechanism of brain edema may be related to its actions: i) high levels of matrix metalloproteinases (MMPs) play a major role in brain edema. Rosell et al (29) reported that MMP-9 was closely related to BBB breakdown during reperfusion injury in cases of human ischemic stroke. Rosenberg and Navratil (30) suggested that the reason for brain edema was MMP-2 overexpression. Ramos-Fernandez et al (31) confirmed that the MMP-9 level was associated with the volume of cerebral infarction, the severity of stroke, hemorrhagic transformation and the functional outcome after an acute stroke; ii) another mechanism was abnormal transformation of water molecules, and water molecule channel proteins: e.g., aquaporins (AQPs) play an important role in brain edema. AQP4 is a two-way water transfer channel protein, and distributes to astrocyte, capillary endothelial cells, ependymal cells and choroid plexus epithelial cells. Papadopoulous and Verkman (32) demonstrated that brain edema in AQP4-null mice was significantly less severe than that of the wild-type mice. Manley et al (33) also found that AQP4-null mice survived more easily than wild-type mice in the experimental model of acute water poisoning. Yang et al (34) found that glial cell AQP4 overexpression in transgenic mice accelerated brain edema and brain swelling. In the present study, we noted that the expression of AQP4 was higher, and brain edema was more severe in the SAH groups, and that atorvastatin reduced AQP4 expression and water 

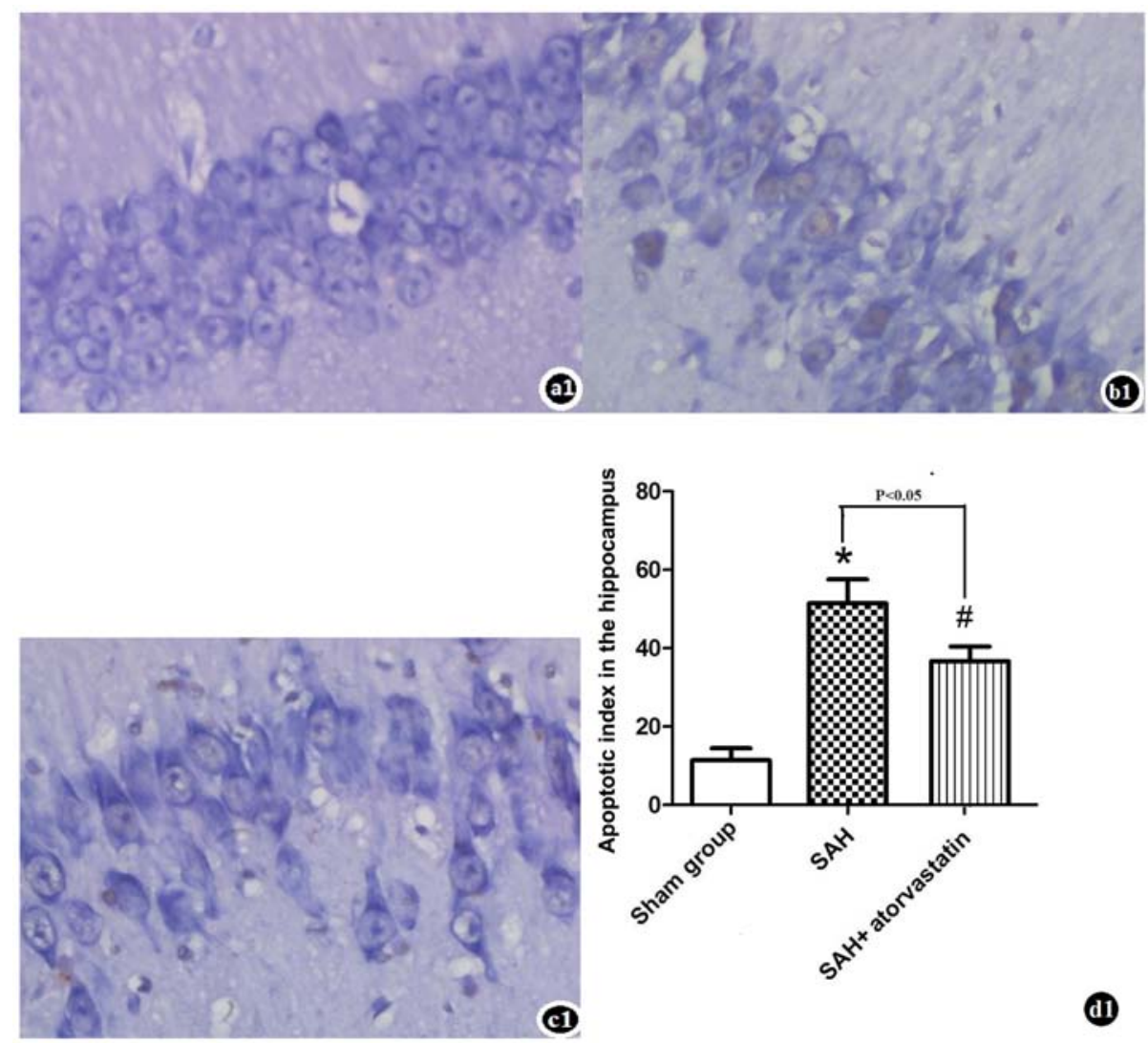

Figure 4. TUNEL staining of basilar artery and basal cortex after subarachnoid hemorrhage (SAH). Few apoptotic cells were observed in sham-operated group rabbits (a1). Increased TUNEL positive staining was observed in SAH rabbits (b1), which was reduced markedly by atorvastatin (c1). Graph showing the apoptotic cells of the groups $(\mathrm{n}=8)$. The bar graph (d1) represents averaged values of the apoptotic cells; data are represented as the means + SD. Sham-operated group vs. SAH group and $\mathrm{SAH}+$ atorvastatin group, ${ }^{*} \mathrm{P}<0.01$ and ${ }^{\#} \mathrm{P}<0.01 ; \mathrm{SAH}+$ atorvastatin group vs. $\mathrm{SAH}$ group, ${ }^{\#} \mathrm{P}<0.05$.

content of the brain tissues. Our results indicated that AQP4 was the key to brain edema, and thus our study provides a new therapeutic target for brain edema.

BBB plays a vital role in the homeostasis of the special internal environment in the central nervous system. The tight junctions of brain microvascular endothelial cells (BMECs) are important in terms of the core structure in the BBB (35). The tight junctions have many important physiological functions and proteins, including occludin, claudins, junction-associated molecules and zonula occludens (36). Researchers have found that occludin interacts closely with other tight junction proteins and they maintain the structure and function of the tight junction complex through the structural domain. Terry et al (37) confirmed that occludin was closely related to the barrier function of BMECs. Huber et al (38) and Persidsky et al (39) confirmed that overexpression of occludin reduced the permeability of the blood brain barrier. Therefore, the tight junction and BMECs maintain the function and reduce the permeability of $\mathrm{BBB}$, and affect cerebral edema. Yi et al (40) found that atorvastatin prevented angiotensin II (Ang II)-induced hyperpermeability and dysregulation of ZO-1 by suppressing Rho kinase (ROCK) signaling, and thus atorvastatin increases the tight junction protein to protect the BBB. Garrido et al (41) also found that atorvastatin protects the BBB and ameliorates brain edema by upregulating claudin 5 , tight junction protein 1. Kalayci et al (42) found that long-term nitric oxide (NOS) inhibition with $\mathrm{N}$ omega-nitro-L-arginine methyl ester (L-NAME) followed by Ang II markedly disrupted the BBB, which may affect CNS homeostasis, and that treatment with atorvastatin improved the perturbations in the BBB by increasing the expression of tight junction proteins.

Atorvastatin inhibits the HMG-CoA reductase $(7,40,42)$. Several studies $(30,43)$ have confirmed that statins significantly inhibit the activity of MMP-2 and MMP-9 to maintain the stability of the BBB. We suggest that atorvastatin ameliorates early brain injury and improves patient prognosis after SAH. Chang et al (26) found that pitavastatin exerts its neuroprotective effect through the dual action of inhibiting cJNK (p46/ p55) activation and reducing cleaved caspase-9a and MMP-9 expression. Zhu et al (44) also demonstrated that simvastatin protected the cerebrum from neuronal excitotoxicity and cytotoxic edema by downregulating the expression of phosphorylated-CaMK II and AQP4 in an animal model of experimental ischemic stroke. Tseng et al (15) first demonstrated that acute treatment with pravastatin after a $\mathrm{SAH}$ was safe and ameliorated CVS, improved cerebral autoregulation, and reduced vasospasm-related delayed ischemic deficits; also, unfavorable outcomes at time of discharge were reduced primarily after aneurysmal SAH in a phase II randomized placebo-controlled trial. Chou et al (20) found that simvastatin for the prevention of delayed cerebral ischemia was safe and feasible after SAH using a randomized placebo-controlled trial containing 39 patients. In the present study, we also observed brain edema and EBI, and demonstrated that atorvastatin ameliorated cerebral edema in a rat SAH model, proving its clinical potential as a treatment strategy to reverse brain edema and EBI in patients suffering from SAH. 

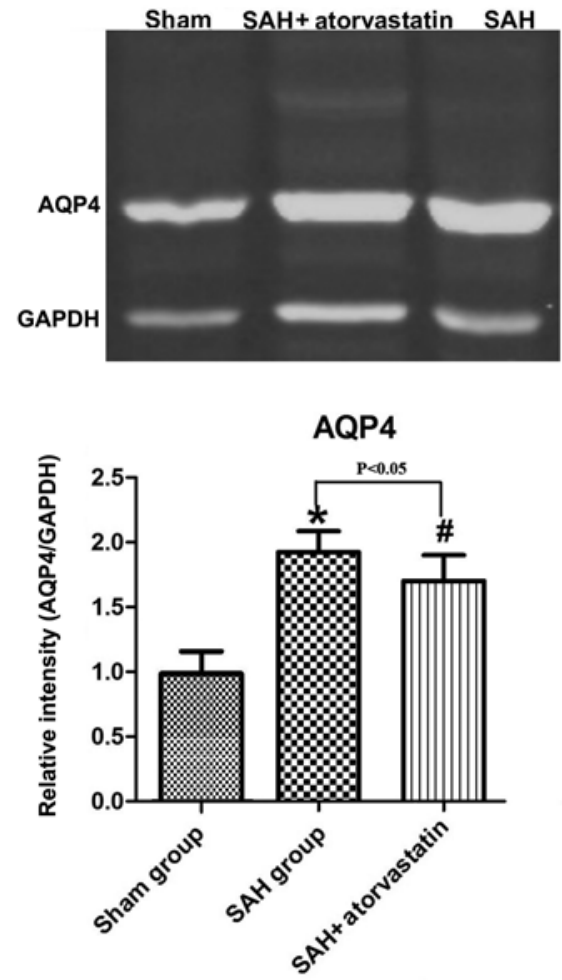

Figure 5. Representative western blot analysis for the expression of aquaporin 4 (AQP4). Graph shows expression levels of AQP4 in the brain cortex of the groups $(n=8)$. Bar graph represents averaged values of AQP4 levels data are represented as the means $+\mathrm{SD}$. Sham-operated group vs. subarachnoid hemorrhage $(\mathrm{SAH})$ group and $\mathrm{SAH}+$ atorvastatin group, ${ }^{*} \mathrm{P}<0.01$ and ${ }^{\#} \mathrm{P}<0.01 ; \mathrm{SAH}+$ atorvastatin group vs. $\mathrm{SAH}$ group, ${ }^{\#} \mathrm{P}<0.05$. Equal protein loading was confirmed by intracellular GAPDH.

Certain studies have reported contradictory results, where statins were not found to have a significant impact on brain edema, delayed cerebral ischemia and brain injury after $\mathrm{SAH}$. One multicenter randomized, controlled, double-blind clinical trial (45) confirmed that high-dose simvastatin or lower doses of simvastatin had no long-term effect on the incidence of delayed ischemic deficits or on the rate of favorable outcomes after SAH. Kirkpatrick et al (23) also reached a similar conclusion: they did not detect any benefit in the use of simvastatin either for the long-term or short-term outcome in patients with SAH in an aneurysmal subarachnoid haemorrhage (STASH) trial.

The mechanism underlying the physiological effects of atorvastatin on sympathetic nerve activity or reversal of over activity, irrespective of whether the sympathetic disorder causes a critical reduction in EBI, can be explained by the relief of brain edema and improvement of outcome. The molecular mechanisms underlying atorvastatin may be related to changes in the expression of two major factors that contribute to brain edema after SAH, AQP4 and ET-1. ET-1 binds to specific receptors on smooth muscle cells and causes constriction of the blood vessels and proliferation of endothelial cells; it exerts deleterious effects on water homeostasis, cerebral edema, and BBB integrity (46). Wang et al (47) confirmed that high levels of ET-1 are closely associated with BBB disruption, and thus it was suggested that it plays an important role in the pathogenesis of secondary brain injury after intracerebral hemorrhage (ICH). Michinaga et al (48) examined the effects
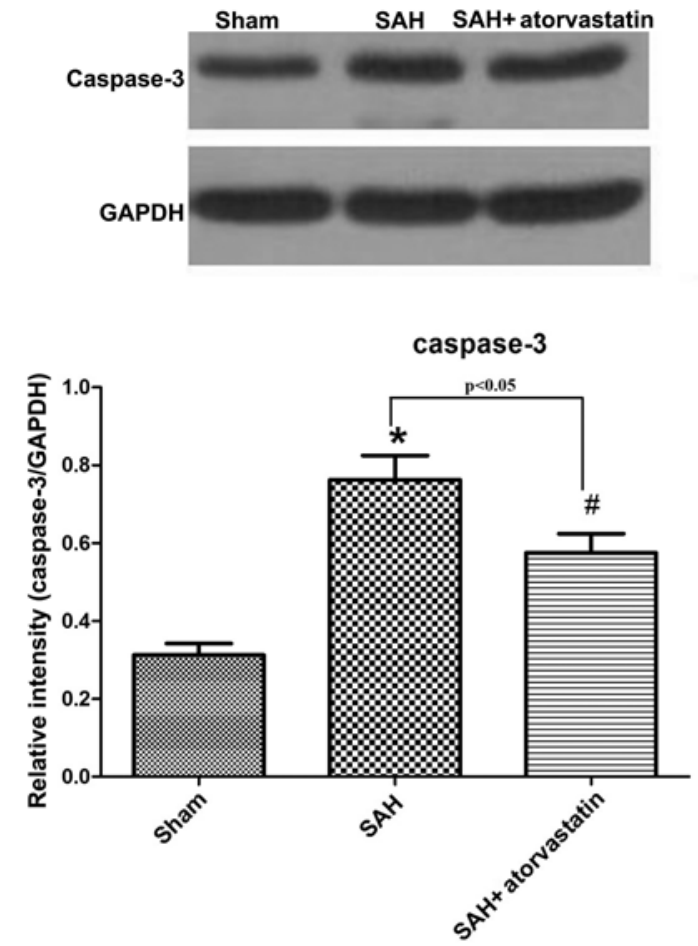

Figure 6. Representative western blot analysis for caspase-3 expression Graph showing the expression levels of caspase-3 in hippocampus of the groups $(n=8)$. Bar graph represents averaged values of the levels of caspase- 3 ; data are represented as the means + SD. Sham-operated group vs. subarachnoid hemorrhage (SAH) group and $\mathrm{SAH}+$ atorvastatin group, ${ }^{*} \mathrm{P}<0.01$ and ${ }^{\#} \mathrm{P}<0.01$. However caspase-3 expression decreased markedly in the $\mathrm{SAH}+$ atorvastatin group vs. $\mathrm{SAH}$ group, ${ }^{\#} \mathrm{P}<0.05$. Equal protein loading was confirmed by intracellular GAPDH.

of ETB antagonists on brain edema formation and disruption of the BBB in a mouse model of cold injury and found that ETB receptor antagonists are important to the amelioration of brain edema. Jo et al (49) also found that endothelial ET-1 expression contributes to epilepticus-induced vasogenic edema formation via BBB disruption in an AQP4/monocyte chemotactic protein 1 (MCP1)-independent manner.

Certain studies (49-51) indicate that the water channel protein AQP4 plays an essential role in water homeostasis and is implicated in the pathogenesis of brain edema. Wang et al (52) found that increased expression of hypoxia-inducible factor $1-\alpha$ (HIF-1 $\alpha$ ) plays a central role in brain edema and BBB disruption by regulating both AQP4 and MMP-9 following experimental SAH. Wang et al (53) studied the functional roles of AQP4 in the different stages of cerebral edema by systematic review; AQP4 may facilitate cerebral edema fluid formation and aggravate the clinical symptoms in cytotoxic edema. It has been suggested that AQP4 is involved in the mechanism of water removal from the interstitial space, ameliorating the level of vasogenic edema as well as intracranial pressure in models of vasogenic edema, such as freezing injury and brain tumors (50).

In the present study, we found that atorvastatin significantly decreased the expression of ET-1 and AQP4 after SAH, possibly resulting in a molecular cascade favoring water removal. This discovery suggests that atorvastatin alleviates brain edema by regulating the plasma concentration of ET-1 and AQP4. However, the precise mechanisms of atorvastatin action need to be studied further. 
It has previously been suggested that apoptosis plays an important role in the long-term morbidity associated with SAH. In the present study, we performed a TUNEL assay to identify and quantify the number of positive cells in the hippocampus of our SAH rabbit model with and without atorvastatin treatment. In our study, we demonstrated that in response to atorvastatin, TUNEL-positive cells were significantly decreased after SAH.

Caspases are a family of cysteine proteases that play an essential role in programmed cell death $(7,54)$. Caspase- 3 is an important member of the caspase family and plays a central role in the execution phase of cell apoptosis. It has previously been reported that the expression of caspase- 3 was increased in brain neurons after SAH and promoted neuron apoptosis (7). In the present study, we analyzed the level of caspase-3 in the hippocampus after SAH. Our data showed that caspase-3 increased significantly in the SAH group but decreased markedly in the $\mathrm{SAH}+$ atorvastatin group. This indicates that atorvastatin activates the anti-apoptotic pathways via the caspase-dependent apoptosis pathway and thus exerts a neuroprotective effect on hippocampal neurons (7), in SAH rabbits.

In conclusion, EBI after SAH is a major contributor to mortality and morbidity. The pathophysiology involves the development of brain edema. Therapeutic options are still limited, as the mechanisms are not fully understood. Our present study provides evidence that atorvastatin may be used for the treatment of cerebral edema and EBI.

\section{References}

1. Komotar RJ, Schmidt JM, Starke RM, Claassen J, Wartenberg KE, Lee K, Badjatia N, Connolly ES Jr and Mayer SA: Resuscitation and critical care of poor-grade subarachnoid hemorrhage. Neurosurgery 64: 397-411, 2009.

2. Rosengart AJ, Schultheiss KE, Tolentino J and Macdonald RL: Prognostic factors for outcome in patients with aneurysmal subarachnoid hemorrhage. Stroke 38: 2315-2321, 2007.

3. Steiner T, Juvela S, Unterberg A, Jung C, Forsting $M$ and Rinkel G: European Stroke Organization guidelines for the management of intracranial aneurysms and subarachnoid haemorrhage. Cerebrovasc Dis 35: 93-112, 2013.

4. Laskowitz DT and Kolls BJ: Neuroprotection in subarachnoid hemorrhage. Stroke 41 (Suppl 10): S79-S84, 2010.

5. MacDonald RL, Higashida RT, Keller E, Mayer SA, Molyneux A, Raabe A, Vajkoczy P, Wanke I, Bach D, Frey A, et al: Clazosentan, an endothelin receptor antagonist, in patients with aneurysmal subarachnoid haemorrhage undergoing surgical clipping: a randomised, double-blind, placebo-controlled phase 3 trail (CONSCIOUS-2). Lancet Neurol 10: 618-625, 2011.

6. Sehba FA, Hou J, Pluta RM and Zhang JH: The importance of early brain injury after subarachnoid hemorrhage. Prog Neurobiol 97: 14-37, 2012.

7. Cheng G, Wei L, Zhi-Dan S, Shi-Guang Z and Xiang-Zhen L: Atorvastatin ameliorates cerebral vasospasm and early brain injury after subarachnoid hemorrhage and inhibits caspase-dependent apoptosis pathway. BMC Neurosci 10: 7, 2009.

8. Budohoski KP, Czosnyka M, Kirkpatrick PJ, Smielewski P, Steiner LA and Pickard JD: Clinical relevance of cerebral autoregulation following subarachnoid haemorrhage. Nat Rev Neurol 9: 152-163, 2013.

9. Uekawa K, Hasegawa Y, Ma M, Nakagawa T, Katayama T, Sueta D, Toyama K, Kataoka K, Koibuchi N, Kawano T, et al: Rosuvastatin ameliorates early brain injury after subarachnoid hemorrhage via suppression of superoxide formation and nuclear factor-kappa B activation in rats. J Stroke Cerebrovasc Dis 23: 1429-1439, 2014.

10. Cahill J, Calvert JW, Solaroglu I and Zhang JH: Vasospasm and p53-induced apoptosis in an experimental model of subarachnoid hemorrhage. Stroke 37: 1868-1874, 2006.
11. Endo H, Nito C, Kamada H, Yu F and Chan PH: Reduction in oxidative stress by superoxide dismutase overexpression attenuates acute brain injury after subarachnoid hemorrhage via activation of Akt/glycogen synthase kinase-3beta survival signaling. J Cereb Blood Flow Metab 27: 975-982, 2007.

12. Aoki K, Zubkov AY, Ross IB and Zhang JH: Therapeutic effect of caspase inhibitors in the prevention of apoptosis and reversal of chronic cerebral vasospasm. J Clin Neurosci 9: 672-677, 2002.

13. Sehba FA, Pluta RM and Zhang JH: Metamorphosis of subarachnoid hemorrhage research: from delayed vasospasm to early brain injury. Mol Neurobiol 43: 27-40, 2011.

14. Tousoulis D, Antoniades C, Katsi V, Bosinakou E, Kotsopoulou M, Tsioufis $\mathrm{C}$ and Stefanadis C: The impact of early administration of low-dose atorvastatin treatment on inflammatory process, in patients with unstable angina and low cholesterol level. Int $\mathbf{J}$ Cardiol 109: 48-52, 2006

15. Tseng MY, Czosnyka M, Richards H, Pickard JD and Kirkpatrick PJ: Effects of acute treatment with pravastatin on cerebral vasospasm, autoregulation, and delayed ischemic deficits after aneurismal subarachnoid hemorrhage: a phase 2 randomized placebo-controlled trial. Stroke 36: 1627-1632, 2005.

16. Lee YM, Chen WF, Chou DS, Jayakumar T, Hou SY, Lee JJ, Hsiao G and Sheu JR: Cyclic nucleotides and mitogen-activated protein kinase: regulation of simvastatin in platelet. J Biomed Sci 17: 45, 2010.

17. Luzak B, Boncler M, Rywaniak J, Wilk R, Stanczyk L, Czyz M, Rysz J and Watala C: The effect of a platelet cholesterol modulation on the acetylsalicylic acid-madiated blood platelet inhibition in hypercholesterolemic patients. Eur J Pharmacol 658: 91-97, 2011.

18. Zhou C, Yamaguchi M, Kusaka G, Schonholz C, Nanda A and Zhang JH: Caspase inhibitors prevent endothelial apoptosis and cerebral vasospasm in dog model of experimental subarachnoid hemorrhage. J Cereb Blood Flow Metab 24: 419-431, 2004.

19. Wang Z, Meng CJ, Shen XM, Shu Z, Ma C, Zhu GQ, Liu HX, He WC, Sun XB, Huo L, et al: Potential contribution of hypoxiainducible factor-1 $\alpha$, aquaporin-4, and matrix metalloproteinase- 9 to blood-brain barrier disruption and brain edema after experimental subarachnoid hemorrhage. J Mol Neurosci 48: 273-280, 2012.

20. Chou SH, Smith EE, Badjatia N, Nogueira RG, Sims JR, Ogilvy CS, RordorfGA and Ayata C: A randomized, double-blind, placebo-controlled pilot study of simvastatin in aneurysmal subarachnoid hemorrhage. Stroke 39: 2891-2893, 2008.

21. Garg K, Sinha S, Kale SS, Chandra PS, Suri A, Singh MM, Kumar R, Sharma MS, Pandey RM, Sharma BS and Mahapatra AK: Role of simvastatin in prevention of vasospasm and improving functional outcome after aneurysmal subarachnoid hemorrhage: a prospective, randomized, double-blind, placebo-controlled pilot trial. Br J Neurosurg 27: 181-186, 2013.

22. Matthew J: Simvastatin for the prevention of symptomatic cerebral vasospasm following aneurysmal subarachnoid hemorrhage: a single-institution prospective cohort study. J Neurosurg 110: 968-974, 2009

23. Kirkpatrick PJ, Turner CL, Smith C, Hutchinson PJ and Murray GD; STASH Collaborators: Simvastatin in aneurysmal subarachnoid haemorrhage(STASH): a multicentre randomised phase 3 trial. Lancet Neurol 13: 666-675, 2014.

24. Vergouwen MD, Meijers JC, Geskus RB, Coert BA, Horn J, Stroes ES, van der Poll T, Vermeulen M and Roos YB: Biologic effects of simvastatin in patients with aneurysmal subarachnoid hemorrhage: a double-blind, placebo-controlled randomized trial. J Cereb Blood Flow Metab 29: 1444-1453, 2009.

25. Chen J, Chen G, Li J, Qian C, Mo H, Gu C, Yan F, Yan W and Wang L: Melatonin attenuates inflammatory response-induced brain edema in early brain injury following a subarachnoid hemorrhage: a possible role for the regulation of pro-inflammatory cytokines. J Pineal Res 57: 340-347, 2014.

26. Chang CZ, Wu SC, Kwan AL and Lin CL: Preconditioning with pitavastatin, an HMG-CoA reductase inhibitor, attenuates C-Jun $\mathrm{N}$-terminal kinase activation in experimental subarachnoid hemorrhage-induced apoptosis. Acta Neurochi 157: 1031-1041, 2015.

27. Broderick JP, Brott TG, Duldner JE, Tomsick T and Leach A: Initial and recurrent bleeding are the major causes of death following subarachnoid hemorrhage. Stroke 25: 1342-1347, 1994.

28. Claassen J, Carhuapoma JR, Kreiter KT, Du EY, Connolly ES and Mayer SA: Global cerebral edema after subarachnoid hemorrhage: frequency, prediction, and impact on outcome. Stroke 33: 1225-1232, 2002. 
29. Rosell A,Cuadrado E, Ortega-Aznar A,Hernandez-Guillamon M, Lo EH and Montaner J: MMP-9-positive neutrophil infiltration is associated to blood-brain barrier breakdown and basal lamina type IV collagen degradation during hemorrhagic transformation after human ischemic stroke. Stroke 39: 1121-1126, 2008.

30. Rosenberg GA and Navratil M: Metalloproteinase inhibition blocks edema in intracerebral hemorrhage in the rat. Neurology 48: 921-926, 1997.

31. Ramos-Fernandez M, Fernanda Bellolio M and Stead LG: Matrix metalloproteinase-9 as a marker for acute ischemic stroke:a systematic review. J Stroke Cerebrovasc Dis 20: 47-54, 2011.

32. Papadopoulous MC and Verkman AS: Aquaporin-4 gene disruption in mice reduces brain swelling and mortality in pneumococcal meningitis. J Biol Chem 280: 13906-13912, 2005.

33. Manley GT, Fujimura M, Ma T, Noshita N, Filiz F, Bollen AW Chan P and Verkman AS: Aquaporin-4 deletion in mice reduces brain edema after acute water intoxication and ischemic stroke. Nat Med 6: 159-163, 2000.

34. Yang B, Zador Z and Verkman AS: G1ial cell aquaporin-4 overexpression in transgenic mice accelerates cytotoxic brain swelling. J Biol Chem 283: 15280-15286, 2008.

35. Abbott NJ, Patabendige AA, Dolman DE Yusof SR and Begley DJ: Structure and function of the blood-brain barrier. Neurobiol Dis 37: 13-25, 2010.

36. Hawkins BT and Davis TP: The blood-brain barrier/neurovascular unit in health and disease. Pharmacol Rev 57: 173-185, 2005.

37. Terry S, Nie M, Matter K and Balda MS: Rho signaling and tight junction functions. Physiology (Bethesda) 25: 16-26, 2010.

38. Huber JD, Egletun RD and Davis TP: Molecular physiology and pathophysiology of tight junctions in the blood-brain barrier. Trends Neurosci 24: 719-725, 2001.

39. Persidsky Y, Ramirez SH, Haorah J and Kanmogne GD: Blood-brain barrier: structural components and function under physiologic and pathologic conditions. J Neuroimmune Pharmacol 1: 223-236, 2006.

40. Yi R, Gao X-P and Hui L: Atorvastatin prevents angiotensin IIinduced high permeability of human arterial endothelial cell monolayers via ROCK signaling pathway. Biochem Biophys Res Commun 459: 94-99, 2015

41. Garrido JM, Esteban M, Roda O, Alaminos M and SánchezMontesinos I: Lysophosphatidic acid pretreatment prevents micromolar atorvastatin-induced endothelial cell death and ensures the beneficial effects of high-concentration statin therapy on endothelial gene expression. Ann Vasc Surg 26: 549-558, 2012.

42. Kalayci R, Kaya M, Elmas I, Arican N, Ahishali B, Uzun H, Bilgic B, Kucuk M and Kudat H: Effects of atorvastatin on blood-brain barrier permeability during 1-NAME hypertension followed by angiotensin-II in rats. Brain Res 1042: 184-193, 2005
43. Seo JH1, Guo S, Lok J, Navaratna D, Whalen MJ, Kim KW and Lo EH: Neurovascular matrix metalloproteinases and the blood-brain barrier. Curr Pharm Des 18: 3645-3648, 2012.

44. Zhu MX, Lu C, Xia CM, Qiao Z and Zhu D: Simvastatin pretreatment protects cerebrum from neuronal injury by decreasing the expressions of phosphor-CaMK II and AQP4 in ischemic stroke rats. J Mol Neurosci 54: 591-601, 2014.

45. Wong GK, Chan DY, Siu DY, Zee BCY, Poon WS, Chan MTV, Gin T and Leung M: High-dose simvastatin for aneurysmal subarachnoid hemorrhage: multicenter randomized controlled double-blinded clinical trial. Stroke 46: 382-388, 2015.

46. Chow M, Dumont AS and Kasselletal NF: Endothelin receptor antagonists and cerebral vasospasm: an update. Neurosurgery 51: 1333-1342, 2002

47. Wang LK, Hong Z, Wu GF and Li C: Perihematomal endothelin-1 level is associated with an increase in blood-brain barrier permeability in a rabbit model of intracerebral hematoma. Chin Med J (Engl) 126: 3433-3438, 2013.

48. Michinaga S, Nagase M, Matsuyama E, Yamanaka D, Seno N, Fuka M, Yamamoto Y and Koyama Y: Amelioration of cold injury-induced cortical brain edema formation by selective endothelin ETB receptor antagonists in mice. PLoS One 9: e102009, 2014.

49. Jo SM, Ryu HJ, Kim JE, Yeo SI, Kim MJ, Choi HC, Song HK and Kang TC: Up-regulation of endothelial endothelin-1 expression prior to vasogenic edema formation in the rat piriform cortex following status epilepticus. Neurosci Lett 501: 25-30, 2011.

50. Manley GT, Binder DK, Papadopoulos MC and Verkman AS: New insights into water transport and edema in the central nervous system from phenotype analysis of aquaporin-4 null mice. Neuroscience 129: 983-991, 2004.

51. Migliati E, Meurice N, DuBois P, Fang JS, Somasekharan S, Beckett E, Flynn G and Yool AJ: Inhibition of aquaporin-1 and aquaporin-4 water permeability by a derivative of the loop diuretic bumetanide acting at an internal pore-occluding binding site. Mol Pharmacol 76: 105-112, 2009.

52. Wang Z, Meng CJ, Shen XM, Shu Z, Ma C, Zhu GQ, Liu HX, He WC, Sun XB, Huo L, et al: Potential contribution of hypoxiainducible factor-1 $\alpha$, aquaporin-4, and matrix metalloproteinase- 9 to blood-brain barrier disruption and brain edema after experimental subarachnoid hemorrhage. J Mol Neurosci 48: 273-280, 2012.

53. Wang WW, Xie C, Zhou LL and Wang GS: The function of aquaporin4 in ischemic brain edema. Clin Neurol Neurosurg 127: $5-9,2014$.

54. Alnemri ES, Livingston DJ, Nicholson DW, Salvesen G, Thomberry NA, Wong WW and Yuan J: Human ICE/CED-3 protease nomenclature. Cell 87: 171, 1996. 